6-8-2021

\title{
A New Generalized Family of Distributions for Lifetime Data
}

Maha A. D. Aldahlan

King Abdulaziz University, maldahlan9999@gmail.com

Mohamed G. Khalil

Benha University

Ahmed Z. Afify

Benha University, ahmed.afify@fcom.bu.edu.eg

Follow this and additional works at: https://digitalcommons.wayne.edu/jmasm

Part of the Applied Statistics Commons, Social and Behavioral Sciences Commons, and the Statistical Theory Commons

\section{Recommended Citation}

Aldahlan, Maha A. D.; Khalil, Mohamed G.; and Afify, Ahmed Z. (2021) "A New Generalized Family of Distributions for Lifetime Data," Journal of Modern Applied Statistical Methods: Vol. 19 : Iss. 1 , Article 6. DOI: $10.22237 / \mathrm{jmasm} / 1608553200$

Available at: https://digitalcommons.wayne.edu/jmasm/vol19/iss1/6

This Regular Article is brought to you for free and open access by the Open Access Journals at DigitalCommons@WayneState. It has been accepted for inclusion in Journal of Modern Applied Statistical Methods by an authorized editor of DigitalCommons@WayneState. 


\section{A New Generalized Family of Distributions for Lifetime Data}

\author{
Maha A. D. Aldahlan \\ King Abdulaziz University \\ Jeddah, Saudi Arabia
}

\author{
Mohamed G. Khalil \\ Benha University \\ Benha, Egypt
}

\author{
Ahmed Z. Afify \\ Benha University \\ Benha, Egypt
}

A new class of continuous distributions called the generalized Burr X-G family is introduced. Some special models of the new family are provided. Some of its mathematical properties including explicit expressions for the quantile and generating functions, ordinary and incomplete moments, order statistics and Rényi entropy are derived. The maximum likelihood is used for estimating the model parameters. The flexibility of the generated family is illustrated by means of two applications to real data sets.

Keywords: $\quad$ Burr $\mathrm{X}$ distribution, generating function, maximum likelihood, order statistic, Rényi entropy

\section{Introduction}

The statistics literature contains many generalized families of distributions which have been proposed through introducing one or more shape parameters to the classical distributions. These families have been extensively and used in modeling data in several applied areas such as engineering, economics, medical sciences and finance, among others. However, applied areas such as finance, lifetime analysis, and insurance require more flexible forms of these distributions.

Gupta et al. (1998) defined the exponentiated-G (exp-G) family by raising the cumulative distribution function $(\mathrm{CDF})$ to a positive power parameter. Some other classes are: the beta-G class presented by Eugene et al. (2002), the KumaraswamyG family introduced by Cordeiro and de Castro (2011), exponentiated generalized$\mathrm{G}$ family proposed by Cordeiro et al. (2013), the Lomax generator due to Cordeiro et al. (2014), the beta transmuted-H family studied by Afify, Yousof, and Nadarajah (2017) and the exponentiated Weibull-H family due to Cordeiro, Afify, Yousof, Pescim, and Aryal (2017).

doi: 10.22237/jmasm/1608553200 | Accepted: Sept. 23, 2018; Published: June 8, 2021.

Correspondence: Ahmed Z. Afify, ahmed.afify @fcom.bu.edu.eg 


\section{ALDAHLAN ET AL}

A new family of distributions is introduced based on the Burr X (BX) distribution. Based on the T-X family pioneered by Alzaatreh et al. (2013), we construct a new generator so-called the generalized Burr X-G (GBX-G) family and give a comprehensive description of some of its mathematical properties.

The probability density function (PDF) and CDF of the BX distribution are given (for $x>0$ ) by

$$
\mathrm{g}(x)=2 \beta x \exp \left(-x^{2}\right)\left[1-\exp \left(-x^{2}\right)\right]^{\beta-1} \text { and } \mathrm{G}(x)=\left[1-\exp \left(-x^{2}\right)\right]^{\beta}
$$

respectively, where $\beta$ is a positive shape parameter.

Let $\mathrm{g}(x ; \boldsymbol{\varphi})$ and $\mathrm{G}(x ; \boldsymbol{\varphi})$ denote the PDF and CDF of the baseline model with parameter vector $\boldsymbol{\varphi}$. Then, the CDF of the GBX-G family becomes

$$
\begin{aligned}
\mathrm{F}(x ; \alpha, \beta, \boldsymbol{\varphi}) & =2 \beta \int_{0}^{\frac{\mathrm{G}(x ; \boldsymbol{\varphi})^{\alpha}}{1-\mathrm{G}(x ; \boldsymbol{\varphi})^{\alpha}}} t \exp \left(-t^{2}\right)\left[1-\exp \left(-t^{2}\right)\right]^{\beta-1} d t \\
& =\left\{1-\exp \left[-\left(\frac{\mathrm{G}(x ; \boldsymbol{\varphi})^{\alpha}}{1-\mathrm{G}(x ; \boldsymbol{\varphi})^{\alpha}}\right)^{2}\right]\right\}^{\beta}
\end{aligned}
$$

The PDF of the GBX-G is given by

$$
\begin{aligned}
\mathrm{f}(x ; \alpha, \beta, \boldsymbol{\varphi})= & \frac{2 \alpha \beta \mathrm{g}(x ; \boldsymbol{\varphi}) \mathrm{G}(x ; \boldsymbol{\varphi})^{2 \alpha-1}}{\left[1-\mathrm{G}(x ; \boldsymbol{\varphi})^{\alpha}\right]^{3}} \exp \left\{-\left[\frac{\mathrm{G}(x ; \boldsymbol{\varphi})^{\alpha}}{1-\mathrm{G}(x ; \boldsymbol{\varphi})^{\alpha}}\right]^{2}\right\} \\
& \times\left\{1-\exp \left[-\left(\frac{\mathrm{G}(x ; \boldsymbol{\varphi})^{\alpha}}{1-\mathrm{G}(x ; \boldsymbol{\varphi})^{\alpha}}\right)^{2}\right]\right\}
\end{aligned}
$$

where $\alpha$ and $\beta$ are positive shape parameters. For $\alpha=1$, the GBX-G family reduces to the Burr X-G (BX-G) class proposed by Yousof et al. (2017). The two additional parameters can allow us to study the tail behavior of the density (2) with more flexibility. Further, the GBX-G class due to its flexibility in accommodating all forms of the hazard rate function (HRF), as shown in the next section, becomes an important class to be used in many applications. 


\section{The GBX-G Special Models}

\section{The GBX-Exponential (GBXEx) Distribution}

Consider the PDF $\mathrm{g}(x)=a e^{-a x}$, for $x>0$, of the exponential (Ex) distribution with positive parameter $a$. Then, the PDF of the GBXEx model is given by

$$
\begin{aligned}
\mathrm{f}(x)= & \frac{2 \alpha \beta a \mathrm{e}^{-a x}\left(1-e^{-a x}\right)^{2 \alpha-1}}{\left[1-\left(1-e^{-a x}\right)^{\alpha}\right]^{3}} \exp \left\{-\left[\frac{\left(1-e^{-a x}\right)^{\alpha}}{1-\left(1-e^{-a x}\right)^{\alpha}}\right]^{2}\right\} \\
& \times\left(1-\exp \left\{-\left[\frac{\left(1-e^{-a x}\right)^{\alpha}}{1-\left(1-e^{-a x}\right)^{\alpha}}\right]^{2}\right\}\right)^{\beta-1}
\end{aligned}
$$

For $\alpha=1$, the GBXEx model reduces to the BXEx model with parameters $\beta$ and $a$. The PDF and HRF plots of the GBXEx model are shown in Figure 1. These plots reveal that the PDF of this model can be reversed J-shape, symmetric, unimodal, left skewed or right skewed. The HRF of the GBXEx distribution can be J-shape, reversed J-shape, decreasing (DFR), increasing (IFR) or bathtub (BT) failure rate shapes.
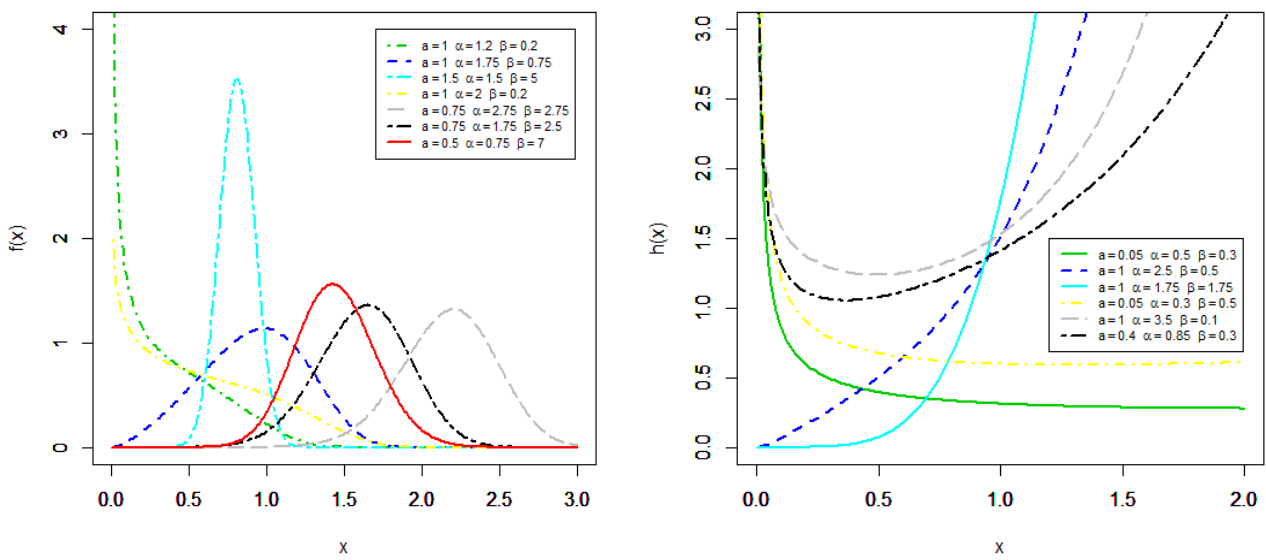

Figure 1. Plots of the PDF and HRF of the GBXEx distribution 


\section{ALDAHLAN ET AL}

\section{The GBX-Weibull (GBXW) Distribution}

Consider the PDF $\mathrm{g}(x)=a b x^{b-1} e^{-a x^{b}}$, for $x>0$, of the Weibull (W) distribution with positive parameters $a$ and $b$. Then, the PDF of the GBXW model is given by

$$
\begin{aligned}
\mathrm{f}(x)= & \frac{2 \alpha \beta a b x^{b-1} e^{-a x^{b}}\left(1-e^{-a x^{b}}\right)^{2 \alpha-1}}{\left[1-\mathrm{G}(x ; \boldsymbol{\varphi})^{\alpha}\right]^{3}} \exp \left\{-\left[\frac{\left(1-e^{-a x^{b}}\right)^{\alpha}}{1-\left(1-e^{-a x^{b}}\right)^{\alpha}}\right]^{2}\right\} \\
& \times\left(1-\exp \left\{-\left[\frac{\left(1-e^{-a x^{b}}\right)^{\alpha}}{1-\left(1-e^{-a x^{b}}\right)^{\alpha}}\right]^{2}\right\}\right)^{\beta-1}
\end{aligned}
$$

Some special cases of the GBXW distribution are: the GBXEx distribution for $b=1$; we obtain the the GBX-Rayleigh (GBXR) distribution for $\beta=2$; the BXW distribution (Yousof et al., 2017) for $\alpha=1$; the BXEx distribution for $\alpha=b=1$; and the BXR model for $\alpha=1$ and $\beta=2$. The plots in Figure 2 show some possible shapes of the PDF and HRF of the GBXW distribution. These plots in Figure 2 show that the PDF of the GBXW can be reversed J-shape, symmetric, left skewed, right skewed, or unimodal shapes. One can see that, the HRF can be Jshape, reversed J-shape, DFR, IFR or BT failure rate shapes.
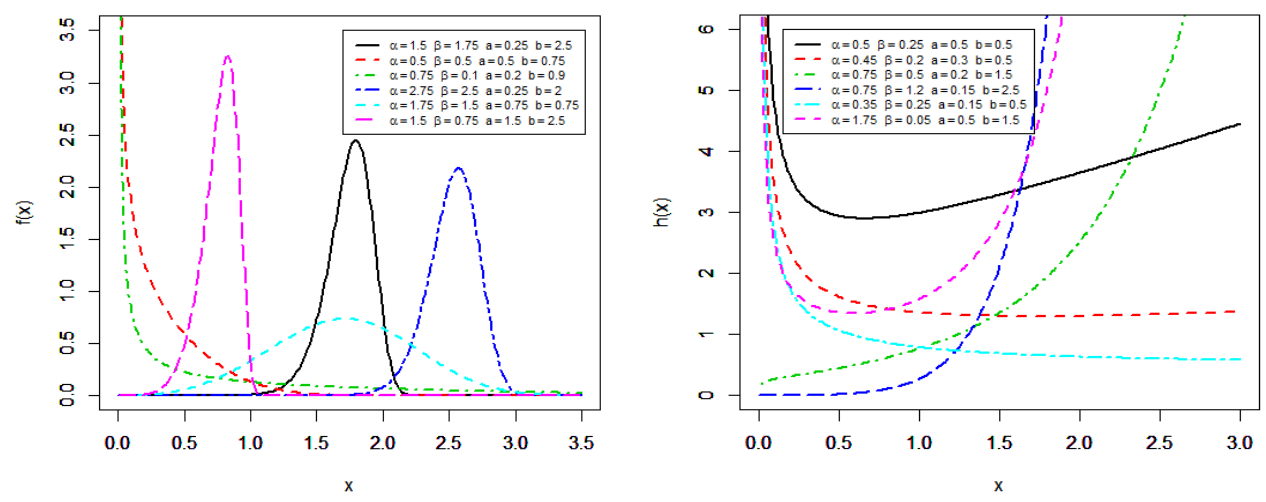

Figure 2. Plots of the PDF and HRF of the GBXW distribution 


\section{GENERALIZED BURR X-G FAMILY}

\section{The GBX-Lomax (GBXLX) Distribution}

The PDF of the Lomax (Lx) distribution with positive parameters $a$ and $b$ is $\mathrm{g}(x)=(a / b)[1+x / b]^{-a-1}$ for $x>0$. Then, the PDF of the GBXLx distribution becomes

$$
\begin{aligned}
\mathrm{f}(x)= & \frac{2 \alpha \beta a\left[1-\left(1-[1+x / b]^{-a}\right)^{\alpha}\right]^{-3}}{b[1+x / b]^{a+1}\left(1-[1+x / b]^{-a}\right)^{1-2 \alpha}} \exp \left\{-\left[\frac{\left(1-[1+x / b]^{-a}\right)^{\alpha}}{1-\left(1-[1+x / b]^{-a}\right)^{\alpha}}\right]^{2}\right\} \\
& \times\left(1-\exp \left\{-\left[\frac{\left(1-[1+x / b]^{-a}\right)^{\alpha}}{1-\left(1-[1+x / b]^{-a}\right)^{\alpha}}\right]^{2}\right\}\right.
\end{aligned}
$$

Plots of the density function of the GBXLx distribution are displayed in Figure 3. Figure 3 shows that the PDF of this model can be reversed J-shape, symmetric, concave down, unimodal, right skewed, or left skewed. The GBXLx HRF can be DFR, IFR, upside down bathtub (UBT), or BT failure rate shapes.
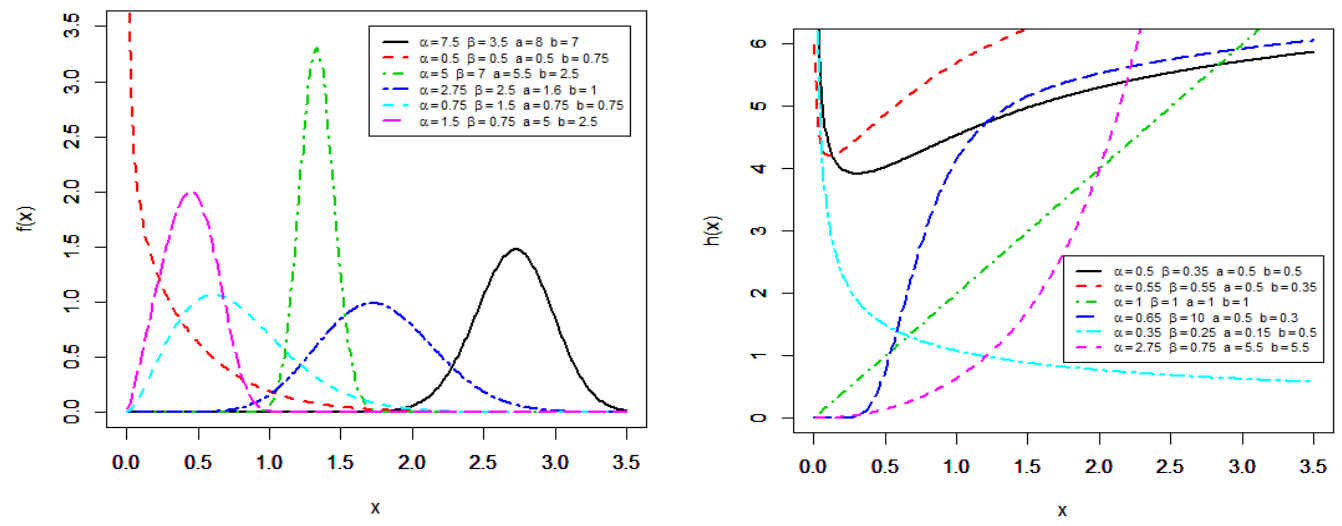

Figure 3. Plots of the PDF and HRF of the GBXLx distribution 


\section{Properties of the GBX-G Family}

\section{Linear Representation}

A useful representation for the GBX-G PDF will now be presented. Consider the power series

$$
(1-z)^{q}=\sum_{l=0}^{\infty}(-1)^{l}\left(\begin{array}{l}
q \\
l
\end{array}\right) z^{l}
$$

By applying (3) to (2),

$$
\mathrm{f}(x)=\frac{2 \alpha \beta \mathrm{g}(x ; \boldsymbol{\varphi}) \mathrm{G}(x ; \boldsymbol{\varphi})^{2 \alpha-1}}{\left[1-\mathrm{G}(x ; \boldsymbol{\varphi})^{\alpha}\right]^{3}} \sum_{l=0}^{\infty}(-1)^{l}\left(\begin{array}{c}
\beta-1 \\
l
\end{array}\right) \exp \left[-\frac{(l+1) \mathrm{G}(x ; \boldsymbol{\varphi})^{2 \alpha}}{\left[1-\mathrm{G}(x ; \boldsymbol{\varphi})^{\alpha}\right]^{2}}\right]
$$

Applying the exponential series to Equation (4),

$$
\mathrm{f}(x)=2 \alpha \beta \mathrm{g}(x ; \boldsymbol{\varphi}) \sum_{l, j=0}^{\infty} \frac{(-1)^{l+j}}{j !(l+1)^{-j}}\left(\begin{array}{c}
\beta-1 \\
l
\end{array}\right) \frac{\mathrm{G}(x ; \boldsymbol{\varphi})^{2 \alpha(j+1)-1}}{\left[1-\mathrm{G}(x ; \boldsymbol{\varphi})^{\alpha}\right]^{2 j+3}}
$$

Consider the series expansion

$$
(1-z)^{q}=\sum_{k=0}^{\infty}(-1)^{k}\left(\begin{array}{c}
-q \\
k
\end{array}\right) z^{k}
$$

Applying the series expansion (5) to Equation (4) gives

$$
\mathrm{f}(x)=2 \alpha \beta \mathrm{g}(x ; \boldsymbol{\varphi}) \sum_{l, j, k=0}^{\infty} \frac{(-1)^{l+j+k}}{j !(l+1)^{-j}}\left(\begin{array}{c}
\beta-1 \\
l
\end{array}\right)\left(\begin{array}{c}
-2 j-3 \\
k
\end{array}\right) \mathrm{G}(x ; \boldsymbol{\varphi})^{\alpha(k+2 j+2)-1}
$$

Thus, the PDF of the GBX-G class reduces to 


\section{GENERALIZED BURR X-G FAMILY}

$$
\mathrm{f}(x)=\sum_{j, k=0}^{\infty} d_{i, j} \mathrm{~h}_{\alpha(k+2 j+2)}(x)
$$

where $\mathrm{h}_{\alpha}(x)=\alpha \mathrm{g}(x) \mathrm{G}(x)^{\alpha-1}$ is the exp-G PDF with power parameter $\alpha$ and

$$
d_{j, k}=2 \beta \sum_{l=0}^{\infty} \frac{(-1)^{l+j+k}(l+1)^{j}}{j !(k+2 j+2)}\left(\begin{array}{c}
\beta-1 \\
l
\end{array}\right)\left(\begin{array}{c}
-2 j-3 \\
k
\end{array}\right) .
$$

Based on Equation (6), several mathematical properties of the GBX-G family can be derived from those of the exp-G family.

\section{Ordinary and Incomplete Moments}

Let $Y_{\alpha(k+2 j+2)}$ denote a random variable having the exp-G distribution with power parameter $\alpha(k+2 j+2)$.

The $r^{\text {th }}$ moment of $X$ follows from (6) as

$$
\mu_{r}^{\prime}=\mathrm{E}\left(X^{r}\right)=\sum_{j, k=0}^{\infty} d_{j, k} \mathrm{E}\left(Y_{\alpha(k+2 j+2)}^{r}\right) .
$$

The $s^{\text {th }}$ incomplete moment of $X$ can be derived from (6) as

$$
\mathrm{m}_{s}(z)=\int_{-\infty}^{z} x^{s} \mathrm{f}(x) d x=\sum_{j, k=0}^{\infty} d_{j, k} \int_{-\infty}^{z} x^{s} \mathrm{~h}_{\alpha(k+2 j+2)}(x) d x
$$

The first incomplete moment of $X$ can be obtained from (7) as

$$
\mathrm{m}_{1}(z)=\sum_{j, k=0}^{\infty} d_{j, k} \mathrm{~J}_{\alpha(k+2 j+2)}(z)
$$

where

$$
\mathbf{J}_{\alpha(k+2 j+2)}(z)=\int_{-\infty}^{z} x^{s} \mathrm{~h}_{\alpha(k+2 j+2)}(x) d x
$$




\section{ALDAHLAN ET AL}

is the first incomplete moment of the exp-G distribution. It can be computed analytically or numerically from the baseline quantile function $(\mathrm{QF}) \mathrm{Q}_{\mathrm{G}}(u)=\mathrm{G}^{-1}(u)$.

\section{Quantile and Generating Functions}

The QF of the GBX-G distribution follows, by inverting (1), as

$$
\mathrm{Q}(u)=\mathrm{Q}_{\mathrm{G}}(u)\left\{\frac{\left[-\ln \left(1-u^{1 / \beta}\right)\right]^{1 / 2}}{1+\left[-\ln \left(1-u^{1 / \beta}\right)\right]^{1 / 2}}\right\}^{\frac{1}{\alpha}},
$$

where $\mathrm{Q}_{\mathrm{G}}(u)=\mathrm{G}^{-1}(u)$ is the $\mathrm{QF}$ of the baseline $\mathrm{G}$ distribution and $u \in(0,1)$.

The moment generating function (MGF) of the GBX-G class follows from Equation (6) as

$$
\begin{aligned}
\mathrm{M}(t) & =\sum_{j, k=0}^{\infty} d_{j, k} \mathrm{M}_{\alpha(k+2 j+2)}(t) \\
& =\sum_{j, k=0}^{\infty} \alpha(k+2 j+2) d_{j, k} \tau(t, \alpha(k+2 j+2)-1)
\end{aligned},
$$

where $\mathrm{M}_{\alpha(k+2 j+2)}(t)$ is the MGF of $Y_{\alpha(k+2 j+2)}$ and

$$
\tau(t, k+j+\alpha-1)=\int_{0}^{1} \exp \left[t \mathrm{Q}_{\mathrm{G}}(u)\right] u^{\alpha(k+2 j+2)-1} d u
$$

Then, the MGF of $X$ can be derived from the exp-G MGF.

\section{Order statistics}

Order statistics are important in several areas of statistical theory and practice. Let $X_{1}, \ldots, X_{n}$ be a random sample from the GBX-G class. The PDF of $X_{i: n}$ is given by

$$
\mathrm{f}_{i: n}(x)=\frac{\mathrm{f}(x)}{\mathrm{B}(i, n-i+1)} \sum_{j=0}^{n-i}(-1)^{j}\left(\begin{array}{c}
n-i \\
j
\end{array}\right) \mathrm{F}(x)^{j+i-1}
$$

where $\mathrm{B}(\cdot, \cdot)$ is the beta function. Based on Equations (1) and (2), one can write 


\section{GENERALIZED BURR X-G FAMILY}

$$
\begin{aligned}
\mathrm{f}(x) \mathrm{F}(x)^{j+i-1}= & \frac{2 \alpha \beta \mathrm{g}(x ; \boldsymbol{\varphi}) \mathrm{G}(x ; \boldsymbol{\varphi})^{2 \alpha-1}}{\left[1-\mathrm{G}(x ; \boldsymbol{\varphi})^{\alpha}\right]^{3}} \exp \left\{-\left[\frac{\mathrm{G}(x ; \boldsymbol{\varphi})^{\alpha}}{1-\mathrm{G}(x ; \boldsymbol{\varphi})^{\alpha}}\right]^{2}\right\} \\
& \times\left\{1-\exp \left[-\left(\frac{\mathrm{G}(x ; \boldsymbol{\varphi})^{\alpha}}{1-\mathrm{G}(x ; \boldsymbol{\varphi})^{\alpha}}\right)^{2}\right]\right\}^{\beta(j+i)-1}
\end{aligned}
$$

Applying the power series (3), the above equation becomes

$$
\begin{aligned}
\mathrm{f}(x) \mathrm{F}(x)^{j+i-1}= & \frac{2 \alpha \beta \mathrm{g}(x ; \boldsymbol{\varphi}) \mathrm{G}(x ; \boldsymbol{\varphi})^{2 \alpha-1}}{\left[1-\mathrm{G}(x ; \boldsymbol{\varphi})^{\alpha}\right]^{3}} \sum_{l=0}^{\infty}(-1)^{l}\left(\begin{array}{c}
\beta(j+i)-1 \\
l
\end{array}\right) \\
& \times \exp \left[-\frac{(l+1) \mathrm{G}(x ; \boldsymbol{\varphi})^{2 \alpha}}{\left[1-\mathrm{G}(x ; \boldsymbol{\varphi})^{\alpha}\right]^{2}}\right]
\end{aligned}
$$

Using the exponential series, we obtain

$$
\mathrm{f}(x) \mathrm{F}(x)^{j+i-1}=2 \alpha \beta \mathrm{g}(x ; \boldsymbol{\varphi}) \sum_{l, s=0}^{\infty} \frac{(-1)^{l+s}}{s !(l+1)^{-s}}\left(\begin{array}{c}
\beta(j+i)-1 \\
l
\end{array}\right) \frac{\mathrm{G}(x ; \boldsymbol{\varphi})^{2 \alpha(s+1)-1}}{\left[1-\mathrm{G}(x ; \boldsymbol{\varphi})^{\alpha}\right]^{2 s+3}} .
$$

After applying the expansion (5), the last equation reduces to

$$
\begin{aligned}
\mathrm{f}(x) \mathrm{F}(x)^{j+i-1}= & \alpha \beta \mathrm{g}(x ; \boldsymbol{\varphi}) \sum_{l, s, k=0}^{\infty} \frac{(-1)^{l+s+k}}{s !(l+1)^{-s}}\left(\begin{array}{c}
\beta(j+i)-1 \\
l
\end{array}\right) \\
& \times\left(\begin{array}{c}
-2 s-3 \\
k
\end{array}\right) \mathrm{G}(x ; \boldsymbol{\varphi})^{\alpha(k+2 s+2)-1}
\end{aligned}
$$

Combining Equations (9) and (10), the PDF of $X_{i: n}$ follows as

$$
\mathrm{f}_{i: n}(x)=\sum_{s, k=0}^{\infty} m_{s, k} \mathrm{~h}_{\alpha(k+2 s+2)}(x),
$$




\section{ALDAHLAN ET AL}

where $\mathrm{h}_{\alpha(k+2 j+2)}(\mathrm{x})$ is the exp-G density with power parameter $\alpha(k+2 s+2)$ and

$$
m_{s, k}=\sum_{l=0}^{\infty} \sum_{j=0}^{n-i} \frac{2 \beta(-1)^{j+l+s+k}(l+1)^{s}}{s !(k+2 s+2) \mathrm{B}(i, n-i+1)}\left(\begin{array}{c}
n-i \\
j
\end{array}\right)\left(\begin{array}{c}
\beta(j+i)-1 \\
l
\end{array}\right)\left(\begin{array}{c}
-2 s-3 \\
k
\end{array}\right)
$$

Hence, the PDF of the GBX-G order statistics is a linear combination of exp-G densities. Equation (11) reveal that the properties of $X_{i: n}$ can be derived from those

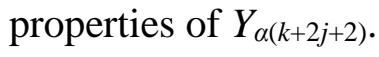

\section{Rényi Entropy}

The Rényi entropy of a random variable $X$ represents a measure of variation of the uncertainty. The Rényi entropy is given by

$$
\mathrm{I}_{\eta}(X)=(1-\eta)^{-1} \log \left(\int_{-\infty}^{\infty} \mathrm{f}(x)^{\eta} d x\right) \quad \eta>0 \quad \text { and } \quad \eta \neq 1
$$

Using the PDF (2),

$$
\begin{aligned}
\mathrm{f}(x)^{\eta}= & \frac{(2 \alpha \beta)^{\eta} \mathrm{g}(x ; \boldsymbol{\varphi})^{\eta} \mathrm{G}(x ; \boldsymbol{\varphi})^{\eta(2 \alpha-1)}}{\left[1-\mathrm{G}(x ; \boldsymbol{\varphi})^{\alpha}\right]^{3 \eta}} \exp \left\{-\eta\left[\frac{\mathrm{G}(x ; \boldsymbol{\varphi})^{\alpha}}{1-\mathrm{G}(x ; \boldsymbol{\varphi})^{\alpha}}\right]^{2}\right\} \\
& \times\left\{1-\exp \left[-\left(\frac{\mathrm{G}(x ; \boldsymbol{\varphi})^{\alpha}}{1-\mathrm{G}(x ; \boldsymbol{\varphi})^{\alpha}}\right)^{2}\right]\right\}^{\eta(\beta-1)}
\end{aligned}
$$

Using the expansion (3),

$$
\begin{aligned}
\mathrm{f}(x)^{\eta}= & \frac{(2 \alpha \beta)^{\eta} \mathrm{g}(x ; \boldsymbol{\varphi})^{\eta} \mathrm{G}(x ; \boldsymbol{\varphi})^{\eta(2 \alpha-1)}}{\left[1-\mathrm{G}(x ; \boldsymbol{\varphi})^{\alpha}\right]^{3 \eta}} \sum(-1)^{l}\left(\begin{array}{c}
\eta(\beta-1) \\
l
\end{array}\right) \\
& \times \exp \left\{-(l+\eta)\left[\frac{\mathrm{G}(x ; \boldsymbol{\varphi})^{\alpha}}{1-\mathrm{G}(x ; \boldsymbol{\varphi})^{\alpha}}\right]^{2}\right\}
\end{aligned}
$$

Using the exponential series and the expansion (5), 


\section{GENERALIZED BURR X-G FAMILY}

$$
\begin{aligned}
\mathrm{f}(x)^{\eta}= & (2 \alpha \beta)^{\eta} \sum_{l, j, k=0}^{\infty} \frac{(-1)^{l+j+k}(l+\eta)^{j}}{j !}\left(\begin{array}{c}
\eta(\beta-1) \\
l
\end{array}\right) \\
& \times\left(\begin{array}{c}
-2 j-3 \eta \\
k
\end{array}\right) \mathrm{g}(x ; \boldsymbol{\varphi})^{\eta} \mathrm{G}(x ; \boldsymbol{\varphi})^{\alpha(2 j+k+2 \eta)-\eta}
\end{aligned}
$$

Then, the Rényi entropy of the GBX-G family reduces to

$$
\mathrm{I}_{\eta}(X)=(1-\eta)^{-1} \log \left[\sum_{j, k=0}^{\infty} w_{j, k} \int_{-\infty}^{\infty} \mathrm{g}(x ; \boldsymbol{\varphi})^{\eta} \mathrm{G}(x ; \boldsymbol{\varphi})^{\alpha(2 j+k+2 \eta)-\eta} d x\right],
$$

where

$$
w_{j, k}=(2 \alpha \beta)^{\eta} \sum_{l=0}^{\infty} \frac{(-1)^{l+j+k}(l+\eta)^{j}}{j !}\left(\begin{array}{c}
\eta(\beta-1) \\
l
\end{array}\right)\left(\begin{array}{c}
-2 j-3 \eta \\
k
\end{array}\right) .
$$

\section{Maximum Likelihood Estimation}

Consider the estimation of the unknown parameters of the GBX-G class from complete samples only by maximum likelihood. Let $X_{1}, \ldots, X_{n}$ be a random sample from this family with parameters $\alpha, \beta$, and $\varphi$. Then, the log-likelihood function of $\boldsymbol{\theta}=\left(\alpha, \beta, \varphi^{\mathrm{T}}\right)^{\mathrm{T}}$, where $\boldsymbol{\theta}$ is the $p \times 1$ parameter vector, is given by

$$
\begin{aligned}
\ell(\boldsymbol{\theta})= & n \log (2 \alpha)+n \log (\beta)+\log \mathrm{g}\left(x_{i} ; \boldsymbol{\varphi}\right)+(2 \alpha-1) \log \mathrm{G}\left(x_{i} ; \boldsymbol{\varphi}\right) \\
& -3 \log \left[1-\mathrm{G}\left(x_{i} ; \boldsymbol{\varphi}\right)^{\alpha}\right]-\left[\frac{\mathrm{G}\left(x_{i} ; \boldsymbol{\varphi}\right)^{\alpha}}{1-\mathrm{G}\left(x_{i} ; \boldsymbol{\varphi}\right)^{\alpha}}\right]^{2} \\
& +(\beta-1) \log \left\{1-\exp \left[-\left(\frac{\mathrm{G}\left(x_{i} ; \boldsymbol{\varphi}\right)^{\alpha}}{1-\mathrm{G}\left(x_{i} ; \boldsymbol{\varphi}\right)^{\alpha}}\right)^{2}\right]\right\}
\end{aligned}
$$

The score vector components,

$$
\mathbf{U}(\boldsymbol{\theta})=\frac{\partial \ell}{\partial \boldsymbol{\theta}}=\left(U_{\alpha}, U_{\beta}, U_{\boldsymbol{\varphi}_{k}}\right)^{\mathrm{T}},
$$




\section{ALDAHLAN ET AL}

are

$$
\begin{aligned}
& U_{\alpha}= \frac{n}{\alpha}+2 \sum_{i=1}^{n} \log \mathrm{G}\left(x_{i} ; \boldsymbol{\varphi}\right)+3 \sum_{i=1}^{n} \frac{\mathrm{G}\left(x_{i} ; \boldsymbol{\varphi}\right)^{\alpha} \log \mathrm{G}\left(x_{i} ; \boldsymbol{\varphi}\right)}{1-\mathrm{G}\left(x_{i} ; \boldsymbol{\varphi}\right)^{\alpha}} \\
&-\sum_{i=1}^{n} \frac{2 \mathrm{G}\left(x_{i} ; \boldsymbol{\varphi}\right)^{2 \alpha}\left[1-2 \mathrm{G}\left(x_{i} ; \boldsymbol{\varphi}\right)^{\alpha}\right] \log \mathrm{G}\left(x_{i} ; \boldsymbol{\varphi}\right)}{\left[1-\mathrm{G}\left(x_{i} ; \boldsymbol{\varphi}\right)^{\alpha}\right]^{3}} \\
&+(\beta-1) \sum_{i=1}^{n} \frac{2\left[1-2 \mathrm{G}\left(x_{i} ; \boldsymbol{\varphi}\right)^{\alpha}\right] \exp \left[-\left(\frac{\mathrm{G}\left(x_{i} ; \boldsymbol{\varphi}\right)^{\alpha}}{1-\mathrm{G}\left(x_{i} ; \boldsymbol{\varphi}\right)^{\alpha}}\right)^{2}\right] \log \mathrm{G}\left(x_{i} ; \boldsymbol{\varphi}\right)}{\left.\left.\left.\mathrm{G}\left(x_{i} ; \boldsymbol{\varphi}\right)^{-2 \alpha}\left[1-\mathrm{G}\left(x_{i} ; \boldsymbol{\varphi}\right)^{\alpha}\right]^{3}\{1-\exp -]^{\mathrm{G}\left(x_{i} ; \boldsymbol{\varphi}\right)^{\alpha}}\right)^{2}\right]\right\}} \\
& U_{\beta}=\frac{n}{\beta}+\sum_{i=1}^{n} \log \left\{1-\exp \left[-\left(\frac{\left.\mathrm{G}\left(x_{i} ; \boldsymbol{\varphi}\right)^{\alpha}\right)^{\alpha}}{1-\mathrm{G}\left(x_{i} ; \boldsymbol{\varphi}\right)^{\alpha}}\right)^{2}\right]\right\}
\end{aligned}
$$

and

$$
\begin{aligned}
& U_{\boldsymbol{\varphi}_{k}}=\sum_{i=1}^{n} \frac{\mathrm{g}^{\prime}\left(x_{i} ; \boldsymbol{\varphi}\right)}{\mathrm{g}\left(x_{i} ; \boldsymbol{\varphi}\right)}+(2 \alpha-1) \sum_{i=1}^{n} \frac{\mathrm{G}^{\prime}\left(x_{i} ; \boldsymbol{\varphi}\right)}{\mathrm{G}\left(x_{i} ; \boldsymbol{\varphi}\right)}+3 \alpha \sum_{i=1}^{n} \frac{\mathrm{G}^{\prime}\left(x_{i} ; \boldsymbol{\varphi}\right)^{\alpha-1}}{1-\mathrm{G}\left(x_{i} ; \boldsymbol{\varphi}\right)^{\alpha}} \\
& -2 \alpha \sum_{i=1}^{n} \frac{\mathrm{G}^{\prime}\left(x_{i} ; \boldsymbol{\varphi}\right)^{\alpha-1}\left\{\left[1-\mathrm{G}\left(x_{i} ; \boldsymbol{\varphi}\right)^{\alpha}\right] \mathrm{G}^{\prime}\left(x_{i} ; \boldsymbol{\varphi}\right)^{\alpha}-\mathrm{G}\left(x_{i} ; \boldsymbol{\varphi}\right)^{2 \alpha}\right\}}{\left[1-\mathrm{G}\left(x_{i} ; \boldsymbol{\varphi}\right)^{\alpha}\right]^{3}} \\
& +(\beta-1) \sum_{i=1}^{n} \frac{\left\{\left[1-\mathrm{G}\left(x_{i} ; \boldsymbol{\varphi}\right)^{\alpha}\right] \mathrm{G}^{\prime}\left(x_{i} ; \boldsymbol{\varphi}\right)^{\alpha}-\mathrm{G}\left(x_{i} ; \boldsymbol{\varphi}\right)^{2 \alpha}\right\} \exp \left[-\left(\frac{\mathrm{G}\left(x_{i} ; \boldsymbol{\varphi}\right)^{\alpha}}{1-\mathrm{G}\left(x_{i} ; \boldsymbol{\varphi}\right)^{\alpha}}\right)^{2}\right]}{\mathrm{G}^{\prime}\left(x_{i} ; \boldsymbol{\varphi}\right)^{1-\alpha}\left[1-\mathrm{G}\left(x_{i} ; \boldsymbol{\varphi}\right)^{\alpha}\right]^{3}\left\{1-\exp \left[-\left(\frac{\mathrm{G}\left(x_{i} ; \boldsymbol{\varphi}\right)^{\alpha}}{1-\mathrm{G}\left(x_{i} ; \boldsymbol{\varphi}\right)^{\alpha}}\right)^{2}\right]\right\}}
\end{aligned}
$$

where 


\section{GENERALIZED BURR X-G FAMILY}

$$
\mathrm{g}^{\prime}\left(x_{i} ; \boldsymbol{\varphi}\right)=\frac{\partial \mathrm{g}\left(x_{i} ; \boldsymbol{\varphi}\right)}{\partial \boldsymbol{\varphi}_{k}} \quad \text { and } \quad \mathrm{G}^{\prime}\left(x_{i} ; \boldsymbol{\varphi}\right)=\frac{\partial \mathrm{G}\left(x_{i} ; \boldsymbol{\varphi}\right)}{\partial \boldsymbol{\varphi}_{k}}
$$

Setting the nonlinear system of equations $U_{\alpha}=U_{\beta}=0$ and $U_{\varphi_{k}}=0$ and solving them simultaneously yields the MLE $\hat{\theta}=\left(\hat{\alpha}, \hat{\beta}, \hat{\boldsymbol{\varphi}}^{\mathrm{T}}\right)^{\mathrm{T}}$. This can be implemented by adopting nonlinear optimization methods such as the quasi-Newton algorithm to maximize $\ell$ numerically.

\section{Applications}

The flexibility of the GBXEx and GBXW distributions will be illustrated using two real data sets. The first data consist of 74 observations on gauge lengths of $20 \mathrm{~mm}$ (Kundu \& Raqab, 2009). The second data set corresponds to the exceedances of flood peaks (in $\mathrm{m}^{3} / \mathrm{s}$ ) of the Wheaton River near Carcross in Yukon Territory, Canada. This data set contains 72 exceedances for the years 1958-1984, rounded to one decimal place (Choulakian \& Stephens, 2001). The GBXEx and GBXW distributions will be compared with some competitive models listed in Table 1. To compare the fitted distributions, we consider some measures namely: Cramér-Von Mises (CVM), Anderson-Darling (AD) and Kolmogorov Smirnov (KS) and its $p$-value (PV). The results in this section are obtained using the R PROGRAM.

Table 1. The competitive models of the GBXEx and GBXW distributions

\begin{tabular}{rr} 
Distribution & Author(s) \\
\hline Exponential (Ex) & Weibull Lindley (WLi) \\
Exponentiated transmuted Burr X (ETBX) & Beta Ex (BEx) \\
Transmuted generalized Ex (TGEX) & Asgharzadeh et al. (2018) \\
Generalized Lindley (GLi) & Afify, Nofal, and Ebraheim (2015) \\
Exponentiated Ex (EEx) & Nadarajah and Kotz (2006a) \\
Alpha power Ex (APEx) & Nhan al. (2017) \\
Weibull (W) & Nadarajah et al. (2011) \\
Kumaraswamy complementary W geometric (KCWG) & Nadarajah and Kotz (2006b) \\
Marshall-Olkin additive W (MOAW) & Mahdavi and Kundu (2016) \\
Alpha logarithmic transformed W (ALTW) & Weibull (1951) \\
Lindley W (LiW) & Afify, Cordeiro, Butt, et al. (2017) \\
Afify, Cordeiro, Yousof, \\
Weibull Burr XII (WBXII) & Saboor, and Ortega (2018) \\
Transmuted complementary W geometric (TCWG) & Cordeiro, Afify, Yousof, \\
Kumaraswamy transmuted W (KTW) & Afify, Cordeiro, Ortega, et al. (2018) \\
Afify, Nofal, and Butt (2014)
\end{tabular}




\section{ALDAHLAN ET AL}

Table 2. MLEs, SEs (in parentheses) and goodness-of-fit measures for gauge lengths data

\begin{tabular}{|c|c|c|c|c|c|c|c|c|}
\hline Model & & Estimate & & & CVM & $A D$ & KS & PV \\
\hline GBXEX & 1.563 & 2.366 & 0.449 & & 0.027 & 0.213 & 0.058 & 0.962 \\
\hline$(\alpha, \beta, a)$ & $(1.221)$ & $(1.810)$ & $(0.194)$ & & & & & \\
\hline LiW & 5.739 & 0.374 & 0.001 & & 0.027 & 0.242 & 0.067 & 0.888 \\
\hline$(\alpha, \beta, \lambda)$ & $(0.508)$ & $(0.007)$ & $(0.063)$ & & & & & \\
\hline ETBX & 24.820 & 0.707 & -0.958 & 0.292 & 0.063 & 0.387 & 0.059 & 0.953 \\
\hline$(\alpha, \beta, \lambda, \delta)$ & $(20.970)$ & $(0.041)$ & $(0.190)$ & $(0.268)$ & & & & \\
\hline BEx & 24.317 & 92.490 & 0.094 & & 0.087 & 0.573 & 0.068 & 0.880 \\
\hline$(a, b, \lambda)$ & (3.988) & $(154.900)$ & $(0.142)$ & & & & & \\
\hline TGEX & 90.150 & 2.215 & -0.697 & & 0.175 & 1.122 & 0.084 & 0.668 \\
\hline$(\alpha, \lambda, \theta)$ & (38.880) & $(0.187)$ & $(0.206)$ & & & & & \\
\hline GLi & 64.830 & 2.291 & & & 0.203 & 1.318 & 0.092 & 0.546 \\
\hline$(\alpha, a)$ & $(23.420)$ & $(0.176)$ & & & & & & \\
\hline EEx & 89.430 & 2.019 & & & 0.217 & 1.405 & 0.095 & 0.512 \\
\hline$(\alpha, \lambda)$ & $(32.470)$ & $(0.171)$ & & & & & & \\
\hline APEx & 1938073.000 & 1.258 & & & 0.115 & 0.752 & 0.191 & 0.008 \\
\hline$(\alpha, \lambda)$ & $(16777.000)$ & $(0.054)$ & & & & & & \\
\hline Ex & 0.403 & & & & 0.087 & 0.574 & 0.449 & 0.000 \\
\hline$(\lambda)$ & $(0.046)$ & & & & & & & \\
\hline
\end{tabular}

Provided in Table 2 are MLEs, their corresponding standard errors (SEs) (in parentheses) and the values of CVM, AD, KS, PV measures for the GBXEx model and other fitted models. Some plots of the fitted GBXEx pdf and other fitted PDFs are displayed in Figure 4. The PP plots of the fitted models are shown in Figure 5. These plots also indicate that the GBXEx model yields the best fit to the glass fibers data compared to the other models.

Provided in Table 3 are the MLEs of the model parameters and the values of CVM, AD, KS, PV statistics for Wheaton River data. The plots of the fitted GBXW density and other fitted densities are displayed in Figure 6. Figure 7 displays the PP plots of the GBXW distribution and other competitive models. These plots reveal that the GBXW distribution yields the best fit and it can be considered a very competitive model to other fitted distributions. 


\section{GENERALIZED BURR X-G FAMILY}
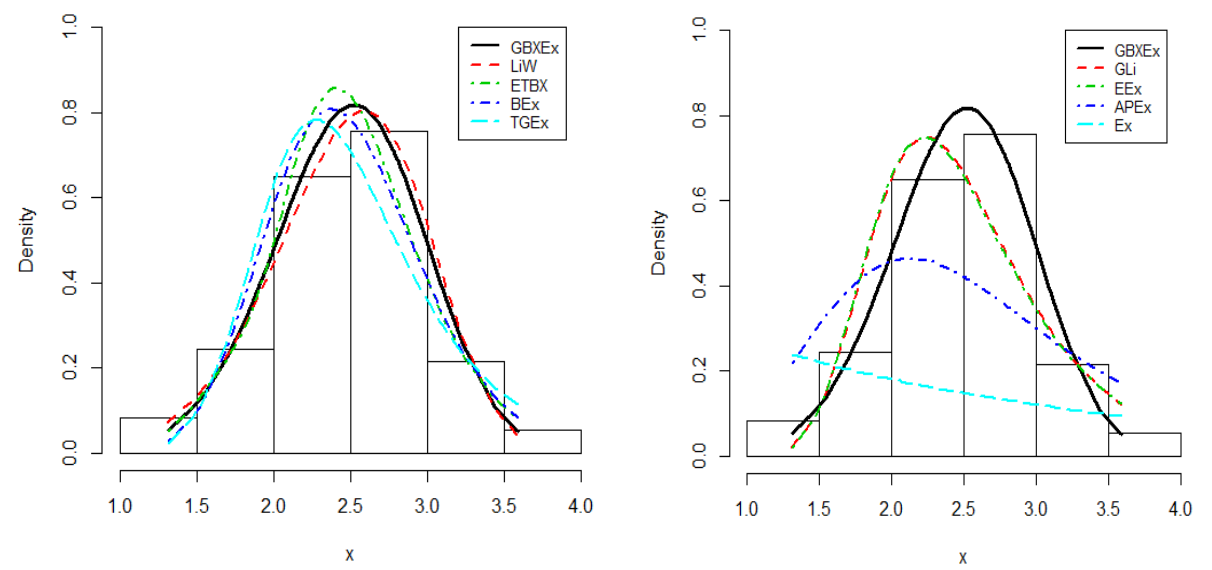

Figure 4. The fitted GBXEx PDF and other PDFs for data set I
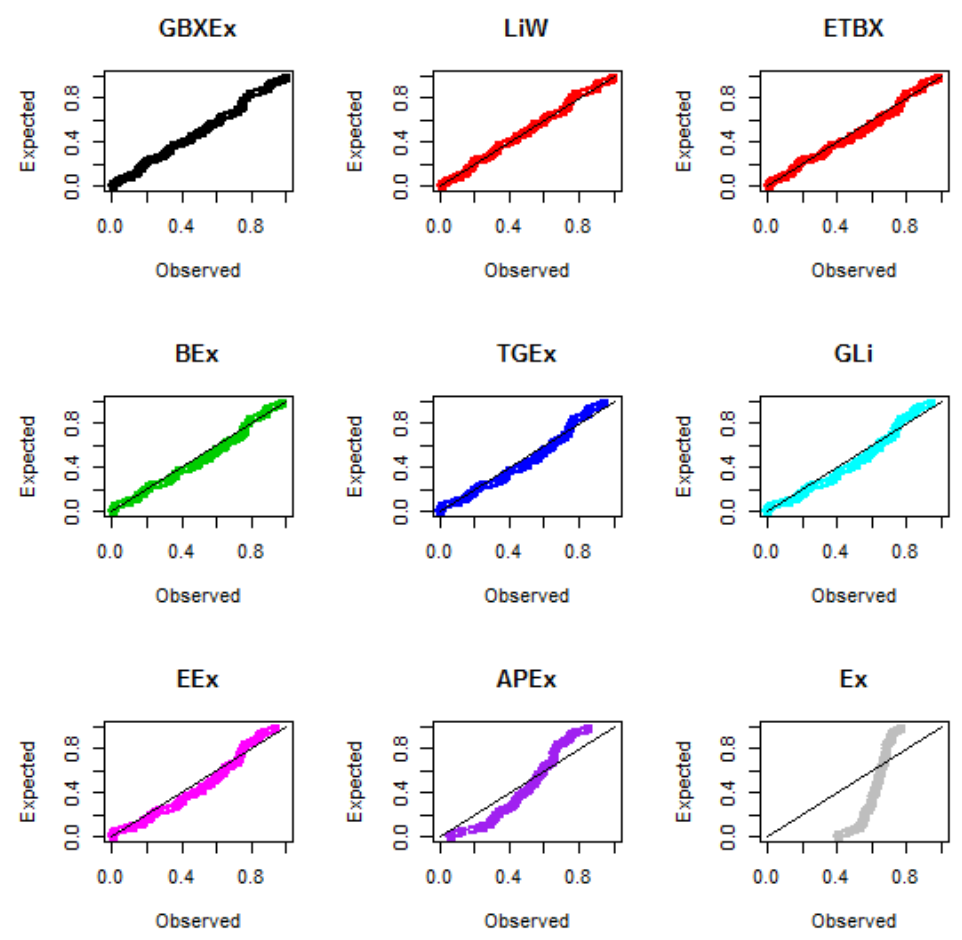

Figure 5. PP plots of the GBXEx distribution and other models for data set I 


\section{ALDAHLAN ET AL}

Table 3. MLEs, SEs (in parentheses), and goodness-of-fit measures for Wheaton river data

\begin{tabular}{rrrrrrrrrr} 
Model & \multicolumn{4}{c}{ Estimates } & & CVM & AD & KS & PV \\
\hline GBXW & 28.162 & 0.217 & 1.824 & 0.207 & & 0.071 & 0.422 & 0.082 & 0.716 \\
$(\alpha, \beta, a, b)$ & $(4.617)$ & $(0.124)$ & $(0.514)$ & $(0.067)$ & & & & & \\
KCWG & 0.236 & 1.258 & 0.025 & 0.612 & 5.077 & 0.094 & 0.577 & 0.100 & 0.466 \\
$(\alpha, \beta, \gamma, a, b)$ & $(1.041)$ & $(1.028)$ & $(0.031)$ & $(0.510)$ & $(20.710)$ & & & & \\
MOAW & 0.002 & 0.725 & 0.150 & 1.191 & 1.714 & 0.101 & 0.623 & 0.099 & 0.473 \\
$(\alpha, \beta, \gamma, \delta, \theta)$ & $(0.002)$ & $(0.195)$ & $(0.217)$ & $(1.755)$ & $(0.265)$ & & & & \\
ALTW & 111.050 & 0.656 & 0.574 & & & 0.100 & 0.636 & 0.111 & 0.332 \\
$(\alpha, \beta, \lambda)$ & $(485.800)$ & $(0.137)$ & $(0.604)$ & & & & & & \\
LiW & 0.042 & 0.868 & 2.406 & & & 0.131 & 0.756 & 0.106 & 0.385 \\
$(\alpha, \beta, \theta)$ & $(0.078)$ & $(0.104)$ & $(3.358)$ & & & & & & \\
WBXII & 0.411 & 1.245 & 0.060 & 1.954 & & 0.133 & 0.766 & 0.106 & 0.391 \\
$(\alpha, \beta, a, b)$ & $(1.041)$ & $(1.179)$ & $(0.214)$ & $(4.651)$ & & & & & \\
TCWG & 0.866 & 0.876 & 0.000 & 0.094 & & 0.135 & 0.776 & 0.107 & 0.38 \\
$(\alpha, \beta, \lambda, \sigma)$ & $(0.812)$ & $(0.168)$ & $(0.445)$ & $(0.051)$ & & & & & \\
KTW & 0.013 & 0.207 & $(0.466)$ & 4.625 & 90.680 & 0.156 & 0.874 & 0.101 & 0.451 \\
$(\alpha, \beta, \lambda, a, b)$ & $(0.016)$ & $(0.135)$ & $(1.619)$ & $(7.303)$ & $(153.800)$ & & & & \\
W & 0.901 & 0.109 & & & & 0.137 & 0.785 & 0.105 & 0.402 \\
$(a, b)$ & $(0.085)$ & $(0.030)$ & & & & & & & \\
\hline
\end{tabular}
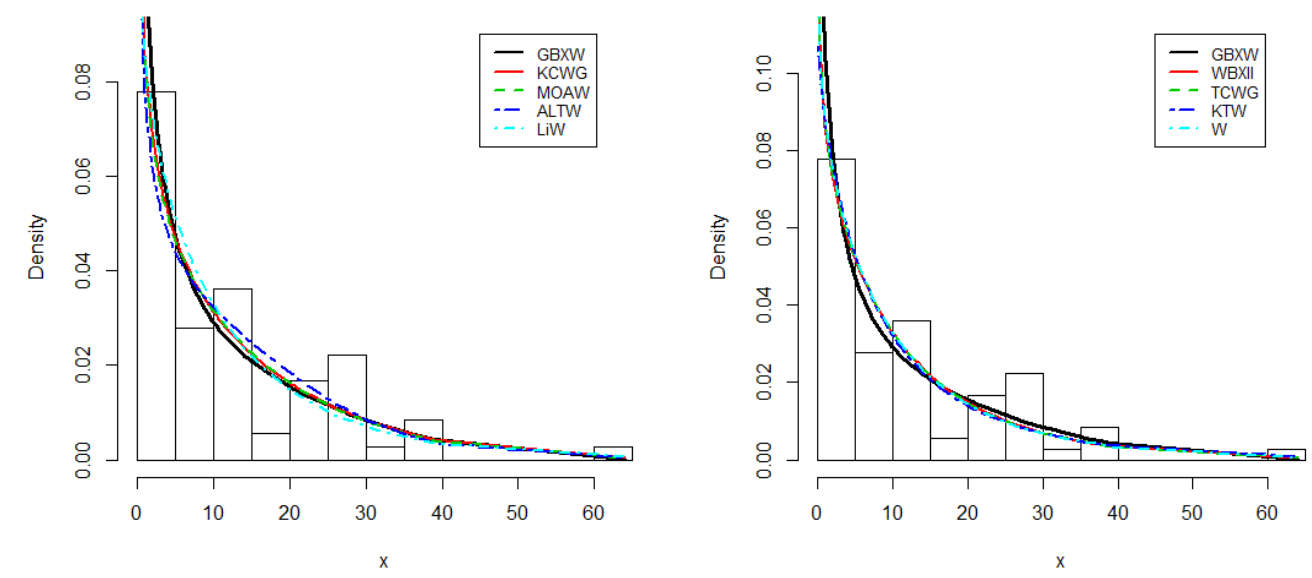

Figure 6. The fitted GBXW PDF and other PDFs for data set II 


\section{GENERALIZED BURR X-G FAMILY}
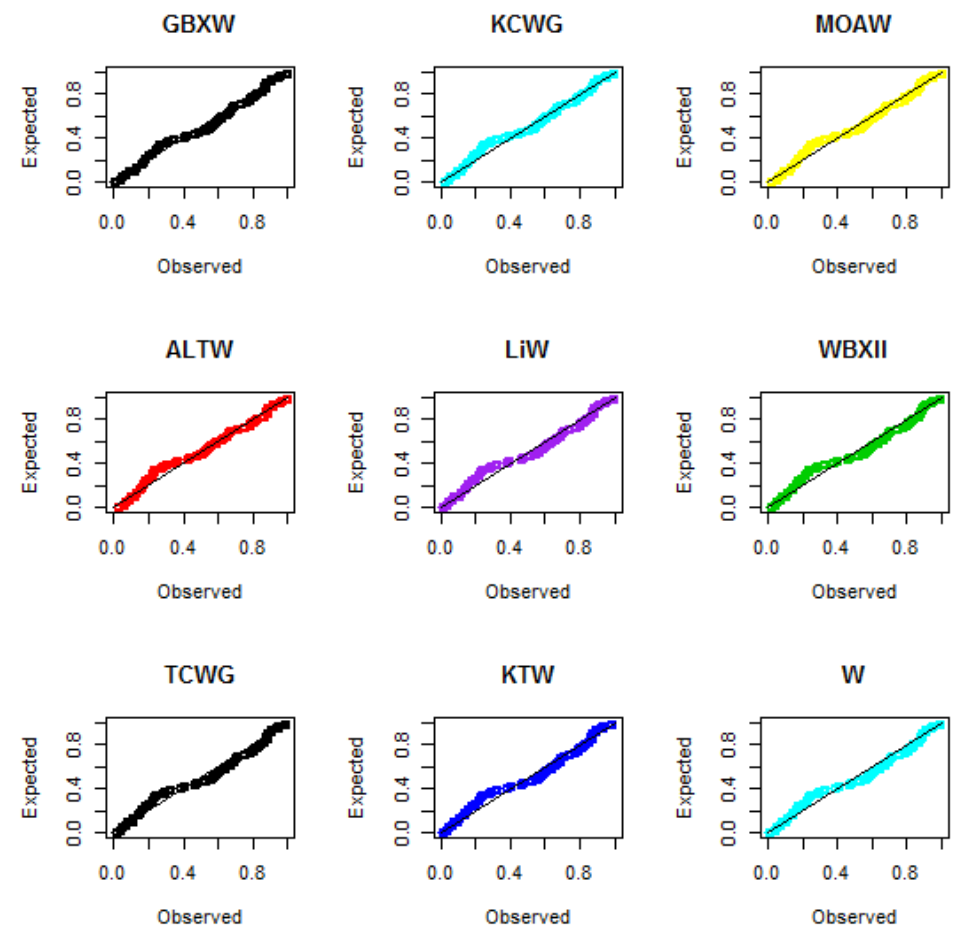

Figure 7. PP plots of the GBXW model and other models for data set II

\section{Simulation}

A Monte Carlo simulation study is provided for the GBXEx model to verify the finite sample behavior of the MLEs of its parameters $a, \alpha$, and $\beta$. There were 1000 samples generated from the GBXEx model using the inversion method with different sample sizes, where $n=20,50,100$, and 200, and with different parameter combinations, where $\quad a=(0.50,0.75), \quad \alpha=(0.50,1.50,250) \quad$ and $\beta=(0.50,0.75,1.75,2.00)$.

Table 4 lists the mean values of estimates, mean square errors (MSE) and bias of the MLEs of the model parameters. The values in Table 4 indicates that the MSEs and biases decrease when the sample size increases. 


\section{ALDAHLAN ET AL}

Table 4. Estimates, bias and MSE of $a, \alpha$, and $\beta$ of the GBXEx distribution

\begin{tabular}{|c|c|c|c|c|}
\hline Parameter & $n$ & Estimate & MSE & Bias \\
\hline$a=0.50$ & 20 & 0.508 & 0.039 & 0.008 \\
\hline$\alpha=1.50$ & & 1.684 & 1.619 & 0.184 \\
\hline \multirow[t]{10}{*}{$\beta=0.50$} & & 0.710 & 0.291 & 0.210 \\
\hline & 50 & 0.495 & 0.018 & -0.041 \\
\hline & & 1.364 & 0.580 & -0.136 \\
\hline & & 0.657 & 0.086 & 0.157 \\
\hline & 100 & 0.437 & 0.010 & -0.063 \\
\hline & & 1.223 & 0.202 & -0.277 \\
\hline & & 0.645 & 0.046 & 0.145 \\
\hline & 200 & 0.430 & 0.007 & -0.070 \\
\hline & & 1.181 & 0.147 & -0.319 \\
\hline & & 0.638 & 0.032 & 0.138 \\
\hline$a=0.75$ & 20 & 0.806 & 0.126 & 0.056 \\
\hline$\alpha=1.50$ & & 2.057 & 3.991 & 0.557 \\
\hline \multirow[t]{10}{*}{$\beta=0.75$} & & 1.040 & 0.585 & 0.290 \\
\hline & 50 & 0.725 & 0.051 & -0.025 \\
\hline & & 1.547 & 0.972 & 0.047 \\
\hline & & 0.946 & 0.193 & 0.196 \\
\hline & 100 & 0.672 & 0.027 & -0.078 \\
\hline & & 1.301 & 0.294 & -0.199 \\
\hline & & 0.948 & 0.115 & 0.198 \\
\hline & 200 & 0.658 & 0.016 & -0.092 \\
\hline & & 1.221 & 0.144 & -0.279 \\
\hline & & 0.930 & 0.055 & 0.180 \\
\hline \multirow{12}{*}{$\begin{array}{l}a=0.75 \\
\alpha=2.50 \\
\beta=1.75\end{array}$} & 20 & 0.767 & 0.037 & 0.017 \\
\hline & & 2.850 & 3.228 & 0.350 \\
\hline & & 2.213 & 1.109 & 0.463 \\
\hline & 50 & 0.718 & 0.015 & -0.032 \\
\hline & & 2.363 & 0.888 & -0.137 \\
\hline & & 2.108 & 0.485 & 0.358 \\
\hline & 100 & 0.702 & 0.008 & -0.048 \\
\hline & & 2.200 & 0.353 & -0.030 \\
\hline & & 2.081 & 0.248 & 0.331 \\
\hline & 200 & 0.695 & 0.005 & -0.055 \\
\hline & & 2.145 & 0.245 & -0.355 \\
\hline & & 2.064 & 0.175 & 0.314 \\
\hline
\end{tabular}




\section{GENERALIZED BURR X-G FAMILY}

Table 4 (continued).

\begin{tabular}{|c|c|c|c|c|}
\hline Parameter & $n$ & Estimate & MSE & Bias \\
\hline$\lambda=0.50$ & 20 & 0.731 & 0.297 & 0.231 \\
\hline$\alpha=0.50$ & & 0.929 & 1.168 & 0.429 \\
\hline$\beta=2.00$ & & 2.395 & 3.564 & 0.395 \\
\hline$\lambda=0.50$ & 50 & 0.605 & 0.081 & 0.105 \\
\hline$\alpha=0.50$ & & 0.645 & 0.193 & 0.145 \\
\hline$\beta=2.00$ & & 2.062 & 1.145 & 0.062 \\
\hline$\lambda=0.50$ & 100 & 0.554 & 0.026 & 0.054 \\
\hline$\alpha=0.50$ & & 0.563 & 0.038 & 0.063 \\
\hline$\beta=2.00$ & & 1.999 & 0.467 & -0.003 \\
\hline$\lambda=0.50$ & 200 & 0.537 & 0.009 & 0.037 \\
\hline$\alpha=0.50$ & & 0.539 & 0.012 & 0.039 \\
\hline$\beta=2.00$ & & 2.040 & 0.178 & 0.040 \\
\hline
\end{tabular}

\section{Conclusions}

In several applied areas, there is a clear need for more flexible distributions which can be used to capture skewness and kurtosis behavior. A new generalized Burr XG (GBX-G) family of distributions was introduced, which extends the Burr X-G class of Yousof et al. (2017) by adding one extra shape parameter. Three special models of the new family were defined. Some mathematical properties of the GBX$\mathrm{G}$ family including explicit expressions for the quantile and generating functions, ordinary and incomplete moments, order statistics, and Rényi entropy are provided. The model parameters are estimated by maximum likelihood. Two real data sets were used to demonstrate certain models of the GBX-G family can provide better fits than other models generated from well-known families.

\section{References}

Afify, A. Z., Cordeiro, G. M., Butt, N. S., Ortega, E. M. M., \& Suzuki, A. K. (2017). A new lifetime model with variable shapes for the hazard rate.

Brazilian Journal of Probability and Statistics, 31(3), 516-541. doi: 10.1214/16BJPS322

Afify, A. Z., Cordeiro, G. M., Ortega, E. M. M., Yousof, H. M., \& Butt, N. S. (2018). The four-parameter Burr XII distribution: properties, regression model and applications. Communications in Statistics - Theory and Methods, 47(11), 2605-2624. doi: 10.1080/03610926.2016.1231821 


\section{ALDAHLAN ET AL}

Afify, A. Z., Cordeiro, G. M., Yousof, H. M., Alzaatreh, A., \& Nofal, Z. M. (2016). The Kumaraswamy transmuted-G family of distributions: properties and applications. Journal of Data Science, 14(2), 245-270. doi:

10.6339/JDS.201604_14(2).0004

Afify, A. Z., Cordeiro, G. M., Yousof, H. M., Saboor, A., \& Ortega, E. M. M. (2018). The Marshall-Olkin additive Weibull distribution with variable shapes for the hazard rate. Hacettepe Journal of Mathematics and Statistics, 47(2), 365381. doi: 10.15672/HJMS.201612618532

Afify, A. Z., Nofal, Z. M., \& Butt, N. S. (2014). Transmuted complementary Weibull geometric distribution. Pakistan Journal of Statistics and Operation Research, 10(4), 435-454. doi: 10.18187/pjsor.v10i4.836

Afify, A. Z., Nofal, Z. M., \& Ebraheim, A. N. (2015). Exponentiated transmuted generalized Rayleigh distribution: A new four parameter Rayleigh distribution. Pakistan Journal of Statistics and Operation Research, 11(1), 115134. doi: 10.18187/pjsor.v11i1.873

Afify, A. Z., Yousof, H. M., \& Nadarajah, S. (2017). The beta transmuted-H family for lifetime data. Statistics and Its Interface, 10(3), 505-520. doi: 10.4310/SII.2017.v10.n3.a13

Alzaatreh, A., Lee, C., \& Famoye, F. (2013). A new method for generating families of continuous distributions. METRON, 71(1), 63-79. doi:

10.1007/s40300-013-0007-y

Asgharzadeh, A., Nadarajah, S., \& Sharafi, F. (2018). Weibull Lindley distribution. REVSTAT - Statistical Journal, 16(1), 87-113.

Choulakian, V., \& Stephens, M. A. (2001). Goodness-of-fit for the generalized Pareto distribution. Technometrics, 43(4), 478-484. doi:

10.1198/00401700152672573

Cordeiro, G. M., Afify, A. Z., Yousof, H. M., Cakmakyapan, S., \& Ozel, G. (2017). The Lindley Weibull distribution: Properties and applications. Anais da Academia Brasileira de Ciências, 90(3), 2579-2598. doi: 10.1590/00013765201820170635

Cordeiro, G. M., Afify, A. Z., Yousof, H. M., Pescim, R. R., \& Aryal, G. (2017). The exponentiated Weibull-H family of distributions: Theory and applications. Mediterranean Journal of Mathematics, 14, 155. doi: 10.1007/s00009-017-0955-1 


\section{GENERALIZED BURR X-G FAMILY}

Cordeiro, G. M., \& de Castro, M. (2011). A new family of generalized distributions. Journal of Statistical Computation and Simulation, 81(7), 883-893. doi: 10.1080/00949650903530745

Cordeiro, G. M., Ortega, E. M. M., \& da Cunha, D. C. C. (2013). The exponentiated generalized class of distributions. Journal of Data Science, 11, 127.

Cordeiro, G. M., Ortega, E. M. M., Popović, B. V., \& Pescim, R. R. (2014). The Lomax generator of distributions: properties, minification process and regression model. Applied Mathematics and Computation, 247, 465-486. doi: 10.1016/j.amc.2014.09.004

Eugene, N., Lee, C., \& Famoye, F. (2002). Beta-normal distribution and its applications. Commun. Stat. Theory Methods, 31, 497-512.

Gupta, R. C., Gupta, P. L., \& Gupta, R. D. (1998). Modeling failure time data by Lehman alternatives. Communications in Statistics - Theory and Methods, 27(4), 887-904. doi: 10.1081/STA-120003130

Khan, M. S., King, R., \& Hudson, I. L. (2017). Transmuted generalized exponential distribution: a generalization of the exponential distribution with applications to survival data. Communications in Statistics - Simulation and Computation, 46(6), 4377-4398. doi: 10.1080/03610918.2015.1118503

Kundu, D., \& Raqab, M. Z. (2009). Estimation of $\mathrm{R}=\mathrm{P}(Y<X)$ for threeparameter Weibull distribution. Statistics and Probability Letters, 79(17), 18391846. doi: 10.1016/j.spl.2009.05.026

Mahdavi, A., \& Kundu, D. (2016). A new method for generating distributions with an application to exponential distribution. Communications in Statistics - Theory and Methods, 46(13), 6543-6557. doi:

10.1080/03610926.2015.1130839

Nadarajah, S., Bakouch, H. S., \& Tahmasbi, R. (2011). A generalized Lindley distribution. Sankhya B, 73, 331-359. doi: 10.1007/s13571-011-0025-9

Nadarajah, S., \& Kotz, S. (2006a). The beta exponential distribution. Reliability Engineering \& System Safety, 91(6), 689-697. doi: 10.1016/j.ress.2005.05.008

Nadarajah, S., \& Kotz, S. (2006b). The exponentiated type distributions. Acta Applicandae Mathematica, 92(2), 97-111. doi: 10.1007/s10440-006-9055-0

Nassar, M., Afify, A. Z., Dey, S., \& Kumar, D. (2018). A new extension of weibull distribution: properties and different methods of estimation. Journal of 


\section{ALDAHLAN ET AL}

Computational and Applied Mathematics, 336, 439-457. doi:

10.1016/j.cam.2017.12.001

Weibull, W. (1951). A statistical distribution function of wide applicability. Journal of Applied Mechanics, 18, 293-297.

Yousof, H. M., Afify, A. Z., Hamedani, G. G., \& Aryal, G. (2017). The Burr $\mathrm{X}$ generator of distributions for lifetime data. Journal of Statistical Theory and Applications, 16(3), 288-305. doi: 10.2991/jsta.2017.16.3.2 